\title{
Context Thesaurus for the Extraction of Metadata from Medical Research Papers
}

\author{
Michael Shepherd, Carolyn Watters, June Young \\ Faculty of Computer Science \\ Dalhousie University \\ Halifax, Nova Scotia, Canada B3H 1 W5 \\ \{shepherd|watters@cs.dal.ca\}
}

\begin{abstract}
Much of the academic literature available on the Web has never been adequately catalogued. Consequently, even using large-scale search engines, much of it remains inaccessible to researchers as indexing on this scale lacks the necessary detail to cope with discipline dependent terminologies and ontologies. Metadata has become a popular means to provide such information within known domains. In this paper, we describe an approach to the automatic extraction of metadata from medical research papers. Medical research papers tend to have stereotypic prescribed sections, such as introduction, methods, and conclusions. The approach described uses context thesauri and the semantic structure of the documents to extract metadata based on these stereotypic sections.
\end{abstract}

\section{Introduction}

"The Semantic Web is not a separate Web but an extension of the current one, in which information is given well-defined meaning, better enabling computers and people to work in cooperation." (3) The first steps towards realizing the Semantic Web are underway with the development and integration of such tools as XML and RDF. If all of the documents on the Web were part of a fully developed Semantic Web and were described with appropriate metadata, then the retrieval of documents relevant to a query would be relatively effective.

Metadata describes the attributes or characteristics of a resource (7) and is seen as a key component of the Semantic Web (10). The association of metadata with documents ranges from simple term occurrence to semantically rich descriptions. Unfortunately, most sophisticated and semantically rich applications of metadata to documents are still handcrafted. The scale of the Web precludes manual assignment of metadata and hence automatic procedures for the extraction of metadata from Web documents are of great interest.

Metadata is often expressed as attribute-value pairs, such as keyword $=$ state or author=Jones, where keyword and author are the attribute names and state and Jones are the attribute values. The attribute-value model of metadata is found in many metadata standards, such as the Dublin Core (8), and there are facilities in the Semantic Web for implementation of this model (2). An attribute may be defined by one or more attribute terms, such as method, measurement, or results. For each such attribute term in a document, an algorithm can be used to determine values for that attribute within that document, i.e., find terms co-occurring with the attribute term. For example, given research as an attribute defining term, co-occurring terms may include \{sports, medical, science, politics\}. If a given document, contains the attribute term, research, and also the term, medical, then we could extract the attributevalue pair, research=medical, for use as metadata for that document. This approach is relatively straightforward to implement but predictably provides low precision.

Context free natural language parsers have also been used on full text documents, such as news, to extract attribute-value pairs with mixed results $(1,4,19)$. Johnston et al. (13) used a domain based parser that recognized keywords and structural relationships in medical documents. Liddy et al. (14) used a natural language parser to generate Dublin Core metadata values for a small set of mathematical education resources with good results compared to manual results. These algorithms are, however, computationally intensive and require domain related dictionaries for good results.

Other researchers have used the structure of the documents to provide some leverage for the generation of better metadata. For example, Giufridda et al. (9) experimented with automatic extraction of metadata based on an analysis of the spatial layout of academic papers. 
Most scientific papers, for example, have the title on the first page in larger letters with authors listed below. Stuckenschmidt and van Harmelen (18) explored using the syntactic structure of Web pages for semi-automatic classification of pages.

In this paper we describe a technique that combines sophisticated term extraction and information about the structure of the documents to generate metadata for research documents using a content thesaurus indexing scheme. We present the results of a preliminary study into the use of context thesauri for this purpose. Section 2 describes the context thesaurus and how we built the context thesaurus from our data set. Section 3 describes the data set while Sections 4 and 5 describe the methodology and results, respectively. Section 6 summarizes this preliminary study outlines future research based on this approach.

\section{Context Thesaurus}

A context thesaurus is an indexing scheme for document collections based on term collocation. At its simplest it is a list of terms related to other terms based on co-occurrence of terms within some window, which could be the entire document. A variety of algorithms have been proposed and used to automatically build such thesauri for use mainly in query expansion $(6,12,17)$.

For example, assume the following collection of four titles:

Doc1: Obesity is a major health problem in the United States.

Doc2: Overweight and obesity are increasing dramatically in the United States.

Doc3: Recent guidelines for treatment of overweight.

Doc4: The increasing prevalence of obesity is a major public health concern.

The list of vocabulary items extracted from these titles may contain Guidelines, Health Concern, Obesity, Overweight, Prevalence, Problem, Treatment, United States. A pseudo-document is generated for each of these vocabulary items consisting of all sentences in which the item occurs. Based on the above vocabulary item list, we have eight pseudo-documents:

\section{Guideline}

Recent guidelines for treatment of overweight.

2. Health concern

The increasing prevalence of obesity is a major public health concern.

3. Overweight

Overweight and obesity are increasing dramatically in the United States.

Recent guidelines for treatment of overweight.

4. Obesity

Obesity is a major health problem in the United

States.

Overweight and obesity are increasing

dramatically in the United States.

The increasing prevalence of obesity is a major public health concern.

\section{Prevalence}

The increasing prevalence of obesity is a major public health concern.

6. Problem

Obesity is a major health problem in the United States.

7. Treatment

Recent guidelines for treatment of overweight.

8. United States

Obesity is a major health problem in the United

States.

Overweight and obesity are increasing dramatically in the United States.

A context thesaurus index is then an inverted list of significant term found in these pseudo-documents. Assume that a partial list of the significant terms found include the terms guideline, health, obesity and overweight. The context thesaurus index for these significant terms is:

Guideline: 1, 3, 7

Health: 2, 4, 5, 6, 8

Obesity: 2, 3, 4, 5, 6, 8

Overweight: $1,3,4,7,8$

The numbers in the above are context thesaurus keys (pseudo-document numbers). If we search for the term overweight, we find that the term occurs in pseudodocuments $1,3,4,7$, and 8 . Therefore, we get a context thesaurus for the term overweight, consisting of the five vocabulary items of the pseudo-documents (guideline, overweight, obesity, treatment, United States). These terms can then be used to refine a query. For instance, if the query term were overweight, we would retrieve the following four documents based on the occurrence of the query term and/or the one or more terms from the query term's context thesaurus. Note that the term overweight occurs in only two of the four documents.

Doc1: obesity, United States

Doc2: overweight, obesity, United States

Doc3: guideline, overweight, treatment

Doc4: obesity

In the current research we are interested in using a context thesaurus to identify terms based on their occurrence within the named semantic structure of the 
document, that are suitable for attribute-value style metadata.

Within disciplines and within genre, document types often follow stereotypical semantic structures such as purpose, method, observation, and conclusion for descriptions of experiments or interventions and outcomes for medical publications. For example, in medicine, "structured abstracts" are used to describe a study using specified content headings rather that paragraph format to help health professionals quickly assess the reliability and content of a clinical report, to facilitate peer review, and to aid accurate indexing and retrieval of reports from computerized databases such as MEDLINE and EMBASE (16). The use of such structured abstracts has also been suggested for the social science literature (11).

A context thesaurus can be used to exploit this structure. Keywords or phrases found in the text can be indexed with reference to their location within the structure of the document, such as, method or observation. Each such structural reference is known as a context. For example, in a document with purpose, method, observation, and conclusion sections, each keyword or phrase in the document could, potentially, have four contexts - one for each of the four sections. The index for the term gallbladder disease could have as a context, purpose. This index can then be used in retrieval to restrict the scope of the terms within the semantic structure of the document or in metadata extraction to generate the attribute-value pair purpose=gallbladder disease.

Purcell, et al., (15) used context models of medical publications for use in conjunction with full-text indexing for retrieval purposes. They showed that the context model significantly improved the precision of retrieval for fixed levels of recall during testing. The contexts were, however, assigned by human indexers who inserted context markup into the text of the document.

In this paper we describe an approach that builds on Purcell's model of contexts that use automatic extraction of contexts within documents for use as metadata to describe the content of medical research papers as well as retrieval.

\section{Data set}

The data set for this research consisted of a collection of sixty medical journal articles from sixteen issues of the Journal of the American Medical Association (JAMA), from 1999 to 2001. Each issue of this journal has a specific theme. The themes for our selected issues are shown in Table 1. The theme medical education was used for three issues, violence and human rights for three issues, and ten other themes were used for the other issues.

Table 1. JAMA themes

\begin{tabular}{ll}
\hline \multicolumn{1}{c}{ JAMA issue } & \multicolumn{1}{c}{ Theme } \\
Feb. 7, 2001 & $\begin{array}{l}\text { Forecasting Opportunities in } \\
\text { Medical research }\end{array}$ \\
March 21, 2001 & Women's Health \\
August 1, 2001 & Violence/Human Rights \\
Sept. 5, 2001 & Medical Education \\
July 12, 2000 & HIV/AIDS \\
August 2, 2000 & Violence/Human Rights \\
August 9, 2000 & Tobacco \\
Sept. 6, 2000 & Medical Education \\
Oct. 25, 2000 & Health Care Systems/Access to \\
& Care \\
Nov. 15, 2000 & End of Life Care \\
May 5, 1999 & Cancer \\
August 4, 1999 & Violence/Human rights \\
Sept. 1, 1999 & Medical Education \\
Oct. 27, 1999 & Obesity Research \\
Nov. 17, 1999 & New Technologies in Medicine \\
Dec. 1, 1999 & Cardiovascular Disease \\
\hline
\end{tabular}

The articles follow a prescribed stereotypic structure as shown in Figure 1, although not all articles contain all of the sections or subsections.

Table 2 shows the list of section titles in the documents of the test collection that were used for the generation of context thesauri. Each of these sections occurred in every document in the test collection. Although the Introduction, Author and References sections did occur in the test collection, context thesauri were not created for these sections. Although each article had introductory text after the abstract and before the next section, there were no explicit Introduction headings and therefore it was not possible to generate a context thesaurus for this heading. No context thesaurus was created for the Author section as names and places can be recognized but not with the algorithms we are investigating. No context thesaurus was created for the References section as we felt it could also be handled better with other algorithms. 


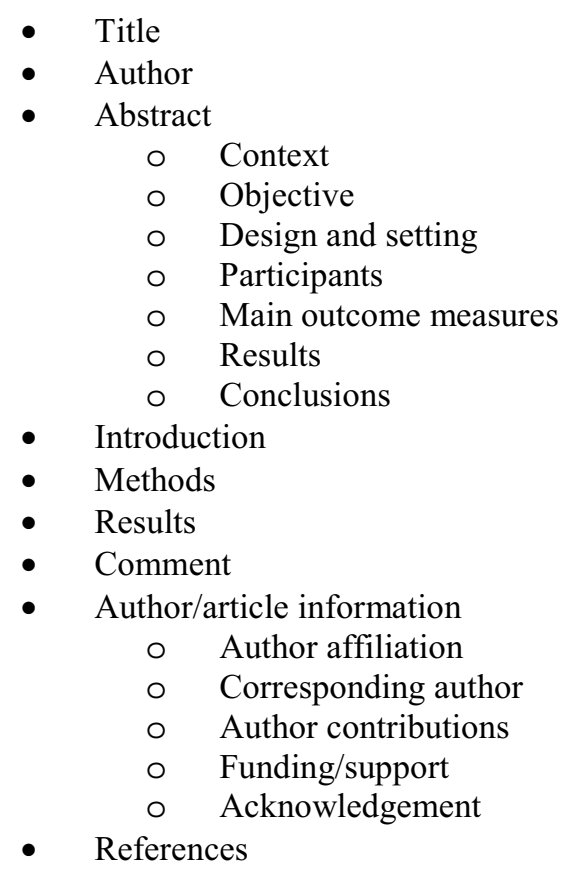

Figure 1. Sections and subsections of the article
structure Table 2. Sections titles used to generate context
thesauri

\begin{tabular}{ll}
\hline \multicolumn{1}{c}{ Attribute } \\
Comment \\
Conclusions \\
Context \\
Design \\
Measures \\
Methods \\
Objective \\
Results \\
\hline
\end{tabular}

\section{Methodology}

The goal of the research is to extract suitable terms from the documents to instantiate appropriate attributevalue pairs for the attributes given in Table 2 that could be used as metadata for the documents. The attributes are meant to reflect the semantic structure of the documents. Therefore, each section title of the eight different sections (Table 2) occurring in the collection is deemed to be an attribute. The task is to extract appropriate values from the documents and associate these values with the appropriate attributes.
The approach taken in this research is based on the creation of context thesauri. We used the KSS suite of tools (5) to create the context thesauri. KSS is a java class library containing objects for manipulating all the information involved in text mining, search and query refinement. This library was developed by the IBM TJ Watson Research Center and contains three parts: the text mining tools, the database management tools and the search tools. The text mining tools consist of a suite of programs for recognizing major terms in a document and aggregating these terms into a collection vocabulary. The Textract program recognizes technical terms, proper names, organizations and places. It also determines named relations between terms using English language patterns such as appositives, parenthetical and comma-separated lists, and unnamed relations based on proximity. Textract gives each term a collection-level importance ranking called the IQ or Information Quotient. IQ is effectively a measure of the document selectivity of a particular term. Textract produces a concordance of all the sentences in the collection that contain these major, salient terms. This concordance may then be used in conjunction with a search engine to create a context thesaurus index of terms that co-occur near a specified term.

There were three different approaches investigated, described in the following subsections.

\subsection{Global Context Thesaurus}

A global context thesaurus was created for the entire document collection of sixty documents, using the KSS libraries. The context window in this instance is the entire document. For example, given the attribute term methods, the KSS system identified the significant terms that occurred in the same documents in which the term methods occurred, based on frequencies within the document and across the collection. The context for each of the eight attribute terms shown in Table 2 was then retrieved from the global thesaurus. This gave all of the significant terms associated with each attribute term, across the entire database.

Those documents in which each of the eight attributes occurred was then searched for the occurrence of these significant terms. The significant terms from step one formed the context across the entire database, yet not every significant term occurred in each document. Therefore, significant terms for each document were identified based on the occurrence of those terms anywhere in that document. For each significant term that occurred, the attribute and the significant term were assigned as attribute-value metadata for that document. Thus, each significant term in a document was associated 
with the attribute terms that occurred in that document. For example, the term NHANES (National Health and Nutrition Examination Survey) occurs in the same document with the attribute terms, design, measures and methods. This generates metadata for that document of, design $=$ NHANES, measures $=$ NHANES, methods $=$ NHANES

\subsection{Section Context Thesaurus}

A section context thesaurus was created in which the contexts were the different sections occurring in the articles. Each document was decomposed into its sections, i.e., measures, methods, objectives, etc. Each section was then treated as a separate document, so that an original document may generate up to eight new partial documents, one for each named section. All of these new partial documents were then grouped by section type (author, measure, etc.) to form eight section-documents. Each section-document consisted of all and only the sections of that type that occurred in the data set. A section context thesaurus for each section-document was then created and used to define metadata for the articles, as was done in steps one and two. At this point, there are two sets of metadata; one set generated from a collection of sixty documents with eight sections per document and the other set generated from a collection of eight documents with up to sixty sections.

\subsection{Focused Global}

Finally, the significant terms identified in the global context thesaurus were passed against the sectiondocument database in order to only pick up those terms that occur in the appropriately named sections. It is focused global in the sense that the context for the creation of the thesaurus is based on the document (identification of significant terms), but the assignment of those terms as metadata values to documents is focused on the occurrence of those terms in the appropriate sections of the documents.

\section{Results}

The global and section context thesauri were generated on different organizations of the collection; a collection of sixty documents with up to eight sections per document versus a collection of eight documents with up to sixty sections per document. Due to this, the contexts of the thesauri generated were quite different and thus the metadata generated was quite different. These differences manifested themselves in the following ways:
- The accuracy of the global approach with the entire document as the context was quite poor,

- The coverage of the attributes was quite different

- The number of values assigned to each attribute differed significantly

Most of these differences were overcome through generating the significant terms from the global context thesaurus but focusing the assignment of terms as metadata only if the terms occurred in the appropriate named section of the document.

\subsection{Accuracy of Global Approach}

Since the metadata values generated from the global context thesaurus are taken from the entire document set based on co-occurrence of the attribute and value term anywhere in the context (document), the proportion of terms occurring in the appropriate section was quite low. As can be seen from Table 3, the proportion of significant terms assigned as metadata to a document that actually occurred in the appropriate section of the document ranged from a low of 0.06 to a high of only 0.34 .

\section{Table 3. Global context thesaurus accuracy}

\begin{tabular}{lc}
\hline \multicolumn{1}{c}{ Attribute } & $\begin{array}{c}\text { Proportion Correct } \\
\text { Section }\end{array}$ \\
\hline Comment & 0.24 \\
Conclusions & 0.06 \\
Context & 0.28 \\
Design & 0.04 \\
Measures & 0.12 \\
Methods & 0.34 \\
Objective & 0.09 \\
Results & 0.25 \\
\hline
\end{tabular}

\subsection{Coverage of Attributes}

The coverage of the attributes differed significantly in the three different approaches (Table 4). The global context approach, with the entire document as the context, generated metadata for every attribute for every document (even if the metadata value did not occur in the correct section). 
Table 4. Coverage of attributes

\begin{tabular}{l|ccc}
\hline \multicolumn{1}{c|}{ Attribute } & Global & Section & Focused Global \\
\hline Comment & 60 & 15 & 25 \\
Conclusions & 60 & 16 & 21 \\
Context & 60 & 11 & 39 \\
Design & 60 & 13 & 35 \\
Measures & 60 & 23 & 53 \\
Methods & 60 & 50 & 52 \\
Objective & 60 & 2 & 22 \\
Results & 60 & 51 & 60 \\
\hline
\end{tabular}

The reorganization of the data into eight section-based documents caused the KSS system to identify a very different set of terms as being significant and thus the resulting context thesaurus was quite different. This resulted in very low coverage of the attributes. Using the section context thesaurus approach, metadata for the objective attribute was generated for only two documents while for the results attribute, metadata was generated fifty-one of the sixty documents. On average, the section context thesaurus approach generated metadata for only twenty-three of the sixty documents over the eight attributes.

Coverage was improved using the focused global approach, as shown in Table 4. By generating attribute terms based on the global context and assigning them as metadata values only if they occurred in the appropriate section, the coverage is better than for the pure section context thesaurus approach. This approach generated metadata for an average of thirty-eight of the sixty documents for the eight attributes. It can also be speculated that the focused global approach was more accurate than the global context thesaurus approach as each metadata value assigned occurred in the appropriate section of the document.

\subsection{Comparison of Assigned Values}

The three approaches assigned quite different sets of terms to be used as metadata values. The global context approach assigned a very large number of values and assigned values when the values did not appear on the appropriate sections. The section context approach generated many fewer metadata values (partly because the organization of the database itself was very different) and did not assign values to all of the attributes. However, those values that were assigned did occur in the appropriate sections. The focused approach identified significant terms using the global context thesaurus but only assigned terms as metadata values if those terms occurred in the appropriately title section of the document.
As an example of the difference in assigned metadata values, Table 5 presents the assigned metadata for the document, The Disease Burden Associated with Overweight and Obesity ${ }^{1}$. Metadata was assigned for all eight attributes using the global context thesaurus but only for the three attributes shown in Table using the section context thesaurus, and the four attributes shown for the focused global approach.

\section{Summary}

In this preliminary study we have generated attributevalue metadata for documents based on their stereotypic semantic structure. Where collections of documents exhibit such stereotypic structure, this structure can be used to facilitate the automatic extraction of metadata in the form of attribute-value pairs. This is based on the assumption that phrases occurring within sections in such documents can be used as values in the metadata. IBM's KSS and GTR tools were used to generate context thesauri both for the entire document collection of sixty JAMA articles and for individual sections of the same documents, e.g., methods or context.

The three approaches used were a global context thesaurus based on the document as context, a section context thesaurus based on the section-type as context, and the global context thesaurus with the focus on the section of the document. The global context thesaurus is substantially larger than the section context thesaurus. This difference is due to the nature of the term selection in the KSS system. Significant terms are selected based on term frequencies within the whole document collection, among other factors. The document set for many of the sections, however, is relatively sparse and results in KSS selecting fewer and often different terms as significant terms in the secton-based collection.

Subjectively, the best results appear to be from generating the global context thesaurus on the entire document set, but only selecting terms as metadata values if they appear in the appropriate sections.

Future research will entail varying the size of the context window from that of a document to a few sentences and performing comparison studies to determine the context window that permits generation of the most appropriate metadata for effective retrieval. Once this has been determined, experiments will be undertaken to

\footnotetext{
${ }^{1}$ Aviva Must, Jennifer Spadano, Eugenie H. Coakley, Alison E. Field, Graham Colditz, and William H. Dietz. The Disease Burden Associated With Overweight and Obesity. JAMA 1999 282: 1523-1529.
} 
compare retrieval based on attribute-value metadata for with retrieval based on simple keyword extraction.

documents based on their stereotypic semantic structure

Table 5. Metadata for Global, Section and Focused Approaches

\begin{tabular}{|c|c|c|c|}
\hline Attribute & Global & Section & Focused Global \\
\hline Context & $\begin{array}{l}\text { School of medicine; } \\
\text { prevalence of obesity; } \\
\text { different pattern; treatment of } \\
\text { overweight; United States }\end{array}$ & United States & United States \\
\hline Measures & $\begin{array}{l}\text { Test; fat distribution; } \\
\text { reliability; estimated } \\
\text { prevalence; fatness; convert; } \\
\text { related mortality; high blood } \\
\text { pressure; high blood } \\
\text { cholesterol level; } \\
\text { Plus } 20 \text { more values }\end{array}$ & Prevelance & $\begin{array}{l}\text { High blood pressure; } \\
\text { high blood cholesterol } \\
\text { level; gallbladder } \\
\text { disease; diabetes } \\
\text { mellitus }\end{array}$ \\
\hline Methods & $\begin{array}{l}\text { Cutoff point; map; PORs; LL; } \\
\text { BMIs; serum cholesterol; } \\
\text { estimated prevalence; cream; } \\
\text { NHANES; ethnic group; high } \\
\text { blood cholesterol level; } \\
\text { representative sample; home } \\
\text { interview; dietary; RR }\end{array}$ & $\begin{array}{l}\text { PORs; serum cholesterol; } \\
\text { lung; normal weight; } \\
\text { NHANES; III; home } \\
\text { interview; smoking status }\end{array}$ & $\begin{array}{l}\text { PORs; serum } \\
\text { cholesterol; NHANES; } \\
\text { ethnic group; high } \\
\text { blood cholesterol } \\
\text { level; home interview }\end{array}$ \\
\hline Result & $\begin{array}{l}\text { Human ; Individuals ; T1 ; } \\
\text { high blood pressure ; United } \\
\text { States ; ethnic group ; } \\
\text { estimated prevalence ; } \\
\text { gallbladder disease ; high } \\
\text { blood cholesterol level ; } \\
\text { Reliability ; T2 ; related } \\
\text { mortality ; cutoff point ; small } \\
\text { number ; diabetes mellitus ; } \\
\text { Examination ; representative } \\
\text { sample ; DM ; convert ; } \\
\text { Weight ; causality ; } \\
\text { prevalence of obesity ; } \\
\text { Mexican Americans ; } \\
\text { Analyses ; weight loss ; } \\
\text { fatness }\end{array}$ & & $\begin{array}{l}\text { Individuals; high blood } \\
\text { pressure; gallbladder } \\
\text { disease; high blood } \\
\text { cholesterol level; T2; } \\
\text { DM; weight }\end{array}$ \\
\hline
\end{tabular}




\section{Acknowledgements}

The authors would like to acknowledge the support of IBM in allowing access to their Textract, KSS and GTR software, used in this research. We would also like to acknowledge the assistance of Dr. James W. Cooper of IBM TJ Watson Research Center in answering our questions about the software. We would also like to thank the Canada Foundation for Innovation for the computer equipment used in this research.

\section{References}

[1] María José Aramburu Cabo and Rafael Berlanga Llavori. "An Approach to a Digital Library of Newspapers", Information Processing and Management, 1997, 33(5): 645-661

[2] Dave Beckett, Eric Miller and Dan Brickley. 2002-0731. Expressing Simple Dublin Core in RDF/XML. Available $\quad 18 \quad$ April 2003 [http://dublincore.org/documents/2002/07/31/dcmes$\mathrm{xml} /]$

[3] T. Berners-Lee, J. Hendler and O. Lassila. "The Semantic Web", Scientific American. May 17, 2001. Available April 18, 2003 [http://www.scientificamerican.com/print_version.cfm ?articleID=00048144-10D2-1C7084A9809EC588EF21]

[4] Christina Carrick and Carolyn R. Watters. "Automatic Association of News Items", Information Processing and Management, 1997, 33(5): 615-632.

[5] James W. Cooper. Knowledge System Server: a Java class library for Text Mining. 2.0.2. 2000. IBM T J Watson Research Center, Hawthorne. NY.

[6] James W. Cooper and R.J. Boyd. "Lexical Navigation: Visually Prompted Query Expansion and Refinement", Proc. of DIGLIB97, 1997. Philadelphia, PA.

[7] L. Dempsey and R. Heery. "Metadata: A Current View of Practice and Issues", Journal of Documentation. 1998, 54(2) 145-72.

[8] Dublin Core Metadata Initiative. Dublin Core Metadata Element Set, Version 1.1: Reference Description. 2003-02-04 Available 18 April 2003 [http://dublincore.org/documents/dces/]

[9] Giovanni Giuffrida, Eddie C. Shek and Jihoon Yang. "Knowledge-Based Meta-Data Extraction for PostScript Files", Proc. of $5^{\text {th }} A C M$ conference on Digital Libraries. San Antonio, Texas. 2000. 77-84.
[10] J. Greenberg, S. Sutton and D.G. Campbell "Metadata: A Fundamental Component of the Semantic Web", Bulletin of the American Society for Information Science and Technology. April/May 2003, 29(4).

[11] James Hartley. "Is it Appropriate to Use Structured Abstracts in Social Science Journals?", Learned Publishing 10 1997, (4) 313-317.

[12] Y. Jing, and W.B. Croft. "An Association Thesaurus for Information Retrieval", Proc. of RIAO 94, 1994, New York, NY. pp. 146-60.

[13] D.B. Johnson, R.K. Taira, A.F. Cardenas and D.R. Aberle. "Extracting Information from Free Text Radiology Reports", International Journal on Digital Libraries. 1997, 1: 297-308.

[14 E.D. Liddy, E. Allen, S. Harwell, S. Corieri, O. Yilmazel, N.E. Ozgencil, A. Diekema, N. McCracken, J. Silverstein and S. Sutton. "Automatic Metadata Generation \& Evaluation", Proc. of ACM SIGIR'02. 11-15 August 2002, Tampere, Finland. 401-2.

[15] G.P. Purcell, G.D. Rennels and E.H. Shortliffe. "Development and Evaluation of a Context-Based Document Representation for Searching the Medical Literature". International Journal on Digital Libraries. 1997, 1:288-296.

[16] Roberta W. Scherer and Barbara Crawley. "MS Reporting of Randomized Clinical Trial Descriptors and Use of Structured Abstracts", JAMA. 15 July 1998; 280(3):269-272.

[17] K. Spark-Jones. "Automatic Keyword Classification for Information Retrieval", 1971. Butterworth, London.

[18] H. Stuckenschmidt and F. van Harmelen. "OntologyBased Metadata Generation from Semi-Structured Information", K-CAP'01. 22-23 October 2001. Victoria, BC., Canada. 163-70.

[19] Carolyn Watters, Wanhong Zheng and Evangelos Milios. "Filtering for Medical News Items", Proc. of ASIST, 18-21 November 2002. Philadelphia, PA. 